\title{
Stereo vision based approach for extracting features from digital holograms
}

\author{
Tomi Pitkäaho ${ }^{1}$ and Thomas J. Naughton ${ }^{2,1}$ \\ ${ }^{1}$ University of Oulu, RFMedia Laboratory, Oulu Southern Institute \\ Vierimaantie 5, 84100 Ylivieska \\ Finland \\ 2 Department of Computer Science, National University of Ireland \\ Maynooth, County Kildare \\ Ireland
}

\section{Introduction}

With digital holography one can record and reconstruct real world threedimensional (3D) objects [1,2]. The recorded interference pattern includes information about both amplitude and phase of a wavefront reflected from or transmitted through the object. However, some of the hologram capture setups pose a problem for the reliable reconstruction of quantitative phase information. This can be because the twin image or noise corrupts the reconstructed phase. In such cases it is usual that only amplitude is reconstructed and used as the basis for metrology. A focus criterion is often applied to this reconstructed amplitude to extract depth information from the sensed 3D scene $[3,4]$. In this paper we present an alternative technique based on applying conventional computer stereo vision algorithms to amplitude reconstructions. We show the effectiveness of our technique using digital holograms of both macroscopic and microscopic real-world 3D objects. We discuss sensitivity to the depth of field of reconstructions, and which hologram capture setups are, and which are not, suitable for the technique.

\section{Method}

From a single digital hologram of a 3D scene, we reconstruct two intensity perspectives [5] of the scene separated by a single known angle, and at a single known reconstruction depth. The angle is chosen so that the scene is approximately centred in each field of view. The stereo disparity [6] between these pairs is used to infer depth information for different regions 
Stereo vision based approach for extracting features from digital holograms 2

in the field of view. We calculate the disparity map as follows. For each pixel in the first perspective image $(\mathrm{x}, \mathrm{y})$ we estimate its corresponding pixel in the second perspective $\left(\mathrm{x}^{\prime}, \mathrm{y}\right)$. In digital holography the epipolar geometry is known a priori, which simplifies the procedure and avoids the rectification stage. Because of this, for horizontally separated perspective positions, we need only look in the horizontal direction for correspondences. The disparity for pixel $(x, y)$ is then difference in pixel locations $\left(x-x^{\prime}\right)$, and the set of disparity values corresponding to each pixel $(x, y)$ is called the disparity space image (DSI).

If two different perspectives $\mathrm{L}$ and $\mathrm{R}$ are given, the disparity map $\mathrm{D}$ will be defined as

$$
D(x, y)= \begin{cases}0, \quad \text { if } \min \left\{\operatorname{argmax} C_{x y}(u)\right\} \leq \tau \\ \min \left\{\operatorname{argmax} C_{x y}(u)\right\}, & \text { otherwise }\end{cases}
$$

where $\mathrm{C}$ is the correlation function used to estimate pixel location $\mathrm{L}(\mathrm{x}, \mathrm{y})$ in R. Function argmax finds the disparity $u$ that maximizes correlation. $\min \{$.$\} returns the minimum of a set. The minimum is used if there is more$ than one correlation peak. $\tau$ is a fixed threshold. For each pixel $(x, y)$ in $\mathrm{L}$, $\mathrm{C}$ is a one-dimensional normalized cross-correlation function defined as

$$
C_{x y}(u)=\frac{\sum_{x^{\prime}} t_{x}\left(x^{\prime}, y\right) \mathrm{f}\left(\mathrm{x}^{\prime}-\mathrm{u}, \mathrm{y}\right)}{\sqrt{\sum_{x^{\prime}} \mathrm{t}_{\mathrm{x}}\left(\mathrm{x}^{\prime}, \mathrm{y}\right)^{2} \sum \mathrm{x}^{\prime} \mathrm{f}\left(\mathrm{x}^{\prime}-\mathrm{u}, \mathrm{y}\right)^{2}}}
$$

where $t_{x}\left(x^{\prime}, \mathrm{y}\right)=L_{x}\left(x^{\prime}, \mathrm{y}\right)-\overline{\mathrm{L}}_{\mathrm{x}, \mathrm{y}}, \mathrm{f}\left(\mathrm{x}^{\prime}-\mathrm{u}, \mathrm{y}\right)=\mathrm{R}\left(\mathrm{x}^{\prime}-\mathrm{u}, \mathrm{y}\right)-\overline{\mathrm{R}}_{\mathrm{y}}$, and $\quad \overline{\mathrm{L}}_{\mathrm{x}, \mathrm{y}}=\frac{1}{2 t+1} \sum_{k=x-t}^{x+t} L(k, y)$ Expression $L_{x}\left(x^{\prime}, y\right)=\operatorname{rect}(x-t, x+t) L\left(x^{\prime}, y\right)$ denotes a $2 \mathrm{t}+1$ sized region of pixels around pixel $\mathrm{L}(x, y)$, that is used to estimate the pixel's corresponding location in R. Finally, $\overline{\mathrm{R}}_{\mathrm{y}}=\frac{1}{\mathrm{~N}} \sum \mathrm{x}^{\prime} \mathrm{R}\left(\mathrm{x}^{\prime}, \mathrm{y}\right)$.

Middlebury College provides stereo pair of images that is used to test our algorithm [7]. Input images, ground truth [7], and output of our algorithm can be seen in Fig. 1. Our algorithm is capable of identifying each of the objects at their different relative depths by using the test image pair. 


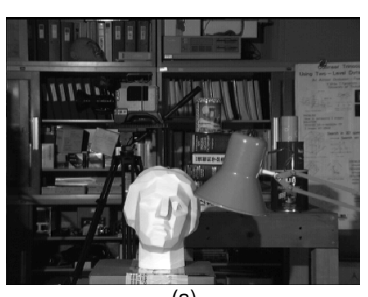

(a)

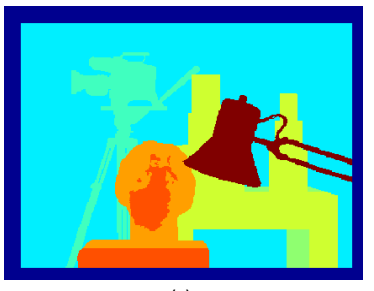

(c)

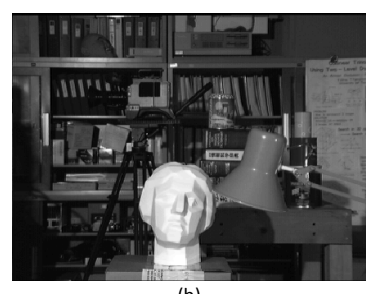

(b)

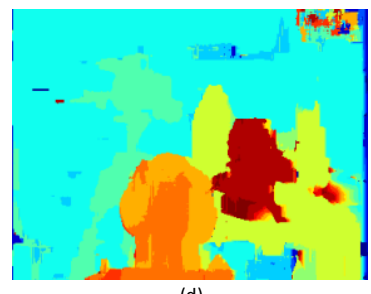

(d)

Fig. 1. Middlebury's test data. (a) left perspective, (b) right perspective, (c) ground truth, (d) output of our algorithm

\section{Microscopic objects}

The first setup used can be seen in Fig. 2. This simple Gabor-type setup consists of a laser, microscopic objective, pinhole, opaque microscopic sample, polarizer, and CCD $(2208 \times 3000$ square pixels with side length 3.5 $\mu \mathrm{m})$. Part of the light going through the sample is diffracted by the sample and creates the object beam, while part of the light goes around the sample undiffracted and creates the reference beam. The object in question is a set of human hairs, whose reconstruction from the whole hologram is shown in Fig. 3.

In Fig. 4 a region of interest from the left and right perspectives can be seen. Figure 4(c) shows the calculated DSI. One quarter of the hologram data was used for each reconstruction, and the depth chosen was the central depth of the scene (between the front and back hairs) In the DSI all three hairs are indentified as are their relative depths: two of the hairs being at the same depth and one of them at a different depth. Quantative measurements of distance were not taken at this stage, but the relative ordering of the objects was verified as being correct. In the colour coding, a whiter colour denotes an object closer to the camera, and a darker colour denotes an object further from the camera. 


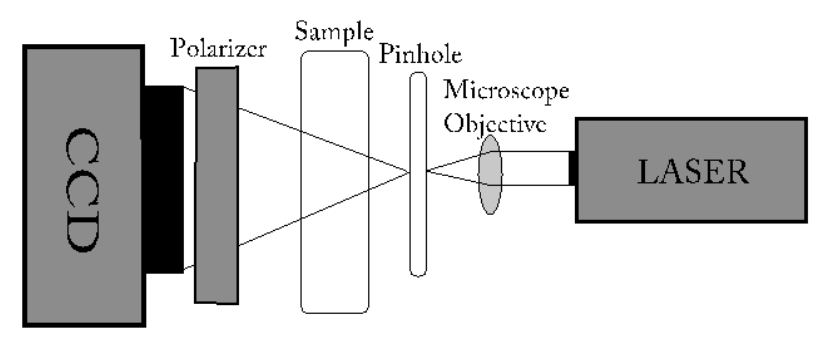

Fig. 2. Microscopic setup

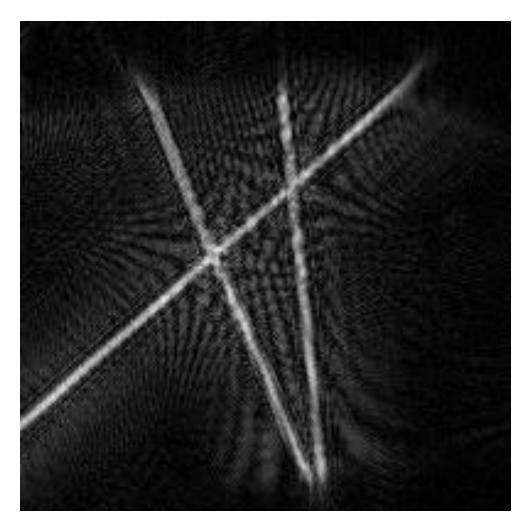

Fig. 3. Human hair reconstruction

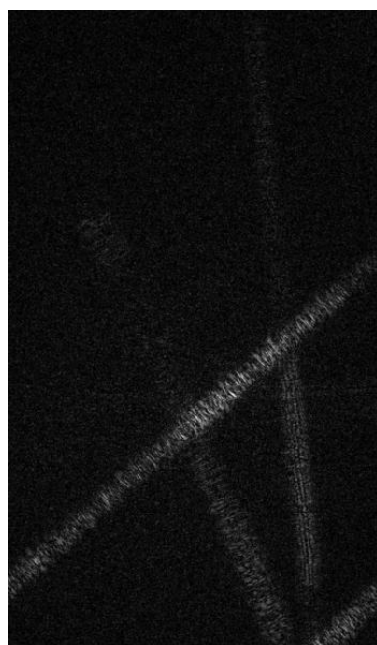

(a)

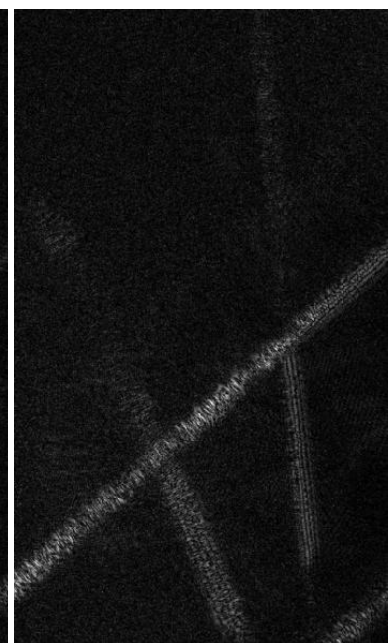

(b)

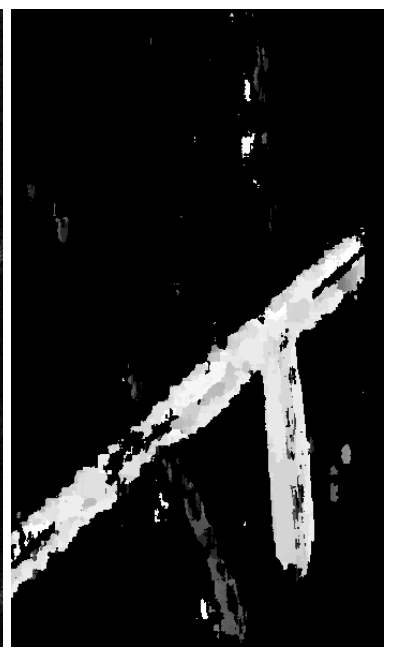

(c)

Fig. 4. Human hair. (a) cropped reconstruction intensity image from left perspective, (b) cropped reconstruction intensity image from right perspective, (c) calculated DSI 
Stereo vision based approach for extracting features from digital holograms 5

\section{Macroscopic objects}

DSI calculations are possible also when using macroscopic real world 3D objects. The setup used is a Mach-Zehnder interferometer architecture. In Fig. 9(a) the intensity of a reconstructed hologram of macroscopic object can be seen. Figure 9(b) shows the calculated DSI, where it can be easily seen which part of the object is located in the front and which is in the back (same colour coding as before). Figures 9 (a) and 9 (b) show the effect of using different amount of hologram data in each reconstruction. Using only a small part of the data gives bigger depth of field, at the expense of reconstruction quality. In DSI calculations a bigger depth of field is usually better because more of the scene is in focus in the reconstructions.

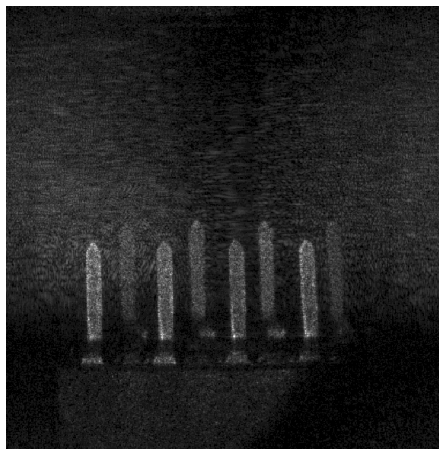

(a)

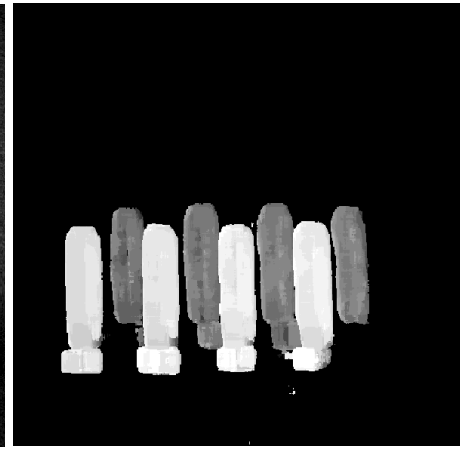

(b)

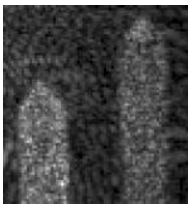

(c)

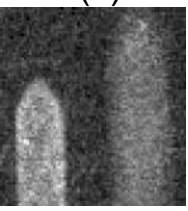

(d)

Fig. 9. Macroscopic object, (a) reconstructed intensity from left perspective using $1 / 4$ of the hologram data, (b) calculated DSI, (c) zoomed part of a, (d) zoomed part when using $1 / 2$ of the hologram data

\section{Conclusions}

We have shown that a traditional computer stereo vision approach can be used in digital holography with different capture setups to obtain depth information of a 3D real-world object. The approach is particularly useful where phase information in the reconstructed domain cannot be readily used to infer depth. Our method uses amplitude reconstruction information only and works well with noisy images, particularly as a result of extending the depth of field, if they have sufficient contrast with their backgrounds. 
Stereo vision based approach for extracting features from digital holograms 6

Both macroscopic and microscopic object hologram capture setups have been show to provide suitable data for our technique. In the microscopic setup case, the applicable setups will be limited to those Gabor type setups where there is sufficient distance between the object and camera. Digital holographic microscope setups whose camera is very close to an image of the observed object are not appropriate for this technique, but they can use reconstructed phase directly for a very accurate quantitative measurement of depth. Our technique could provide clear advantages where one's optical setup does not allow reliable reconstructed phase.

\section{Acknowledgments}

We thank Emmanouil Darakis for capturing the digital holographic data. This project was supported by the Academy of Finland, Science Foundation Ireland, a European Commission Marie Curie Inter-European Fellowship, and the European Community's Seventh Framework Programme FP7/2007-2013 under grant agreement no. 216105.

\section{References}

1. Kreis, T (2005) Handbook of Holographic Interferometry. WileyVHC, Berlin.

2. Schnars, U, Jueptner, W, (2004) Digital Holography: Digital Hologram Recording, Numerical Reconstruction and Related Techniques, Springer.

3. Ma, L, Wang, H, Li, Y, Jin, H (2004) Numerical reconstruction of digital holograms for three-dimensional shape measurement. Journal of Optics A: Pure and Applied Optics, 6, 396-400.

4. McElhinney, C, Hennelly, B, Naughton T, (2008) Extended focused imaging for digital holograms of macroscopic threedimensional objects. Applied Optics, 47, D71-D79.

5. Javidi, B, Tajahuerce, E (2000) Three-dimensional object recognition by use of digital holography. Optics Letters, 25, 610612.

6. Barnard, S, T, Fischler, M, A, (1982) Computational stereo, ACM Computing Surveys, 14(4), 553-572.

7. Scharstein, D, Szelisk, R, (2002) A taxonomy and evaluation of dense two-frame stereo correspondence algorithms. International Journal of Computer Vision, 47, 7-42. 